VoL. 53 (1996) [209-212]

\title{
A NOTE ON HALL CLOSURE OF METANILPOTENT FITTING CLASSES
}

\author{
MaRTin MENTh
}

Metanilpotent Lockett classes are Hall closed. There is an example of a supersoluble, not Hall closed Fitting class.

Let $\mathfrak{F}$ be a Fitting class, that is a class of finite soluble groups that is closed with respect to forming normal subgroups and normal products. If $\pi$ is a set of primes, $\mathfrak{F}$ is said to be Hall- $\pi$-closed provided that whenever $H$ is a Hall- $\pi$-subgroup of $G$ and $G \in \mathfrak{F}$, then $H \in \mathfrak{F}$. The class $\mathfrak{F}$ is said to be Hall-closed if it is Hall- $\pi$-closed for all sets of primes. If $G$ is a finite soluble group, we denote by $G_{\mathfrak{F}}$ the join of all normal $\mathfrak{F}$-subgroups of $G$. A Fitting class $\mathfrak{F}$ satisfying $(G \times H)_{\mathfrak{F}}=G_{\mathfrak{F}} \times H_{\mathfrak{F}}$ for all finite soluble groups $G$ and $H$ is called a Lockett class. For a Fitting class $\mathfrak{F}$ there is a uniquely determined smallest Lockett class $\mathfrak{F}^{*} \supseteq \mathfrak{F}$. The intersection of all Fitting classes $\mathfrak{X}$ with $\mathfrak{X}^{*}=\mathfrak{F}^{*}$ is denoted by $\mathfrak{F}_{*}$. A Fitting class $\mathfrak{F}$ is said to be a Fischer class if for every normal subgroup $K$ of $G \in \mathfrak{F}$ and nilpotent subgroup $H / K$ of $G / K$ we have $H \in \mathfrak{F}$.

Imposing an additional condition for nilpotent length of a Fitting class $\mathfrak{F}$ sometimes yields further closure properties of $\mathfrak{F}$. For instance, every metanilpotent Fischer class is subgroup closed $[5,3.7]$. By weakening the hypothesis 'Fischer class' to 'Lockett class' we cannot expect subgroup closure, but we have:

THEOREM. Every metanilpotent Lockett class is Hall-closed.

Proof: Let $\mathfrak{F}$ be a metanilpotent Fitting class that is not Hall-closed. There exists a set $\pi$ of primes and a group $G \in \mathfrak{F}$ such that $G$ has a Hall- $\pi$-subgroup $H \notin \mathfrak{F}$. Set $F=F i t(G)$, and let $p_{1}, \ldots, p_{n}$ be the prime divisors of $|F|$. Then $F$ is the direct product of its Sylow- $p_{i}$-subgroups $P_{i}, 1 \leqslant i \leqslant n$, and $G / F$ is nilpotent. Having numbered the primes suitably, there is an integer $k(1 \leqslant k<n)$ such that $p_{1}, \ldots, p_{k}$ are the $p_{i}$ in $\pi$. Then $P=P_{1} \cdot \ldots \cdot P_{k}=H \cap F$. The quotient $H / P$ is isomorphic to a subgroup of $G / F$ and therefore nilpotent. So $H / P$ is a subnormal product of cyclic groups $\left\langle x_{i} P\right\rangle$. At least one of the subgroups $\left\langle P, x_{i}\right\rangle$ of $H$ is not an $\mathfrak{F}$-group, let us say $H^{*}=\langle P, x\rangle$. Now we may replace $G$ by $G^{*}=\langle F, x\rangle$, because $G^{*} \in \mathfrak{F}$ and

Received 27th April, 1995

Copyright Clearance Centre, Inc. Serial-fee code: 0004-9729/96 \$A2.00+0.00. 
$H^{*}$ is a Hall- $\pi$-subgroup of $G^{*}$. Set $Q=P_{k+1} \cdot \ldots \cdot P_{n}$. We define a direct product $D=\left\langle P, x_{1}\right\rangle \times\left\langle Q, x_{2}\right\rangle$, where $\left\langle P, x_{1}\right\rangle$ is a copy of $H^{*}$ and $\left\langle Q, x_{2}\right\rangle$ is a copy of $Q\langle x\rangle$. Then $K=P Q\left\langle x_{1} x_{2}\right\rangle$ is a normal subgroup of $D$ and isomorphic to $G^{*}$, so $K \leqslant D_{\mathfrak{F}}$. On the other hand, $x_{1} \notin\left\langle P, x_{1}\right\rangle_{\mathfrak{F}}$. Hence $D_{\mathfrak{F}} \neq\left\langle P, x_{1}\right\rangle_{\mathfrak{F}} \times\left\langle Q, x_{2}\right\rangle_{\mathfrak{F}}$, and $\mathfrak{F}$ is not a Lockett class.

Brison $[1,6.4]$ proved that for an arbitrary Fitting class $\mathfrak{F}$, the class $\mathfrak{F}_{*}$ is Hall closed if and only if $\mathfrak{F}^{*}$ is Hall closed. So we get:

COROLlaRY $1 . \mathfrak{F}^{*}$ and $\mathfrak{F}_{*}$ are Hall closed for every metanilpotent Fitting class $\mathfrak{F}$.

For every supersoluble Fitting class $\mathfrak{F}$, the Lockett closure $\mathfrak{F}^{*}$ is supersoluble $[\mathbf{4}$, 1.1]. Therefore we obtain from the preceeding theorem:

Corollary 2. Every supersoluble Fitting class is a subclass of a supersoluble Hall-closed Fitting class.

The following example shows that not all supersoluble Fitting classes are Hall closed. We need some notation.

Let $p$ be a prime, $p \equiv 1 \bmod 3$ and $n$ a primitive third root of unity in $G F(p)$. Set $T_{p}=\left\langle a, b \mid a^{p}=b^{p}=[a, b, a, a]=[a, b, a, b]=[a, b, b, b]=1\right\rangle \quad$ and $U_{p}=\left\langle T, s \mid s^{3}=1, a^{s}=a^{n}, b^{s}=b^{n}\right\rangle$. The Fitting class $\mathfrak{U}_{p}$ generated by $U_{p}$ can be described in the following way: Let $\mathfrak{U}_{p}^{0}$ be the class of all finite groups $G=X Y$, where $X=O_{p}(G)$ and $Y \in S y l_{3}(G)$, such that

(i) $X$ is a central product of copies $P_{i}$ of $T_{p}$;

(ii) $Y / C_{Y}\left(P_{i}\right) \cong C_{3}$ and $P_{i} Y / C_{Y}\left(P_{i}\right) \cong U_{p}$ for all indices $i$.

Then $\mathfrak{U}_{p}$ is the class of all groups $G \in \mathfrak{S}_{p} \mathfrak{S}_{3}$ such that $O^{p}(G) \in \mathfrak{U}_{p}^{0}$. So $\mathfrak{U}_{p}$ is a Fitting class of 'Dark type', and a supersoluble Lockett class (see [3] and [4]).

EXAMPLE 1. Set $A=T_{7}$ and $B=T_{13}$, and construct a semidirect product $K=$ $(A \times B) \rtimes\langle x\rangle$ in the following way: $x$ raises all elements of $A$ modulo $A^{\prime}$ to the power of 2 and all elements of $B$ modulo $B^{\prime}$ to the power of 3 . That means $\langle A, x\rangle \cong U_{7}$ and $\langle B, x\rangle \cong U_{13}$. Then the Fitting class $\mathfrak{K}$ generated by $K$ has the following properties:

(a) $\mathfrak{K}$ is a supersoluble Fitting class;

(b) the Hall- $\{7,3\}$-subgroups and Hall- $\{13,3\}$-subgroups of $K$ are not in $\mathfrak{K}$.

Proof: The class $\mathfrak{K}$ can be described in the same way as $\mathfrak{U}_{p}$, replacing $T$ by $P=A \times B$ and $U$ by $K$. Denote $X=O_{\{7,13\}}(G)$ and define $\mathfrak{K}^{0}$ in the same way as $\mathfrak{U}_{p}^{0}$. Then $\mathfrak{K}$ is the class of all groups $G \in \mathfrak{N}_{\{7,13\}} \mathfrak{S}_{3}$ such that $O^{\{7,13\}}(G) \in \mathfrak{K}^{0}$. To prove this one can use the same steps as in [3], replacing $T$ by $P$. In particular $\mathfrak{K}$ is supersoluble. If $G$ is a $\{7,13\}$-perfect group in $\mathfrak{K}$ then $O_{\{7,13\}}(G)$ is a central product of $m$ copies of $A$ and of the same number of copies of $B$. The subgroup $A\langle x\rangle$ of $K$ is 
a Hall- $\{7,3\}$-subgroup of $K$, and $B\langle x\rangle$ is a Hall- $\{13,3\}$-subgroup of $K$. Both of them are $\{7,13\}$-perfect, so they are not $\mathfrak{K}$-groups.

Since Fischer classes are Lockett classes, an example of Brison (proof of [1, Proposition 3.3.a]) shows that the theorem cannot be generalized by dropping the metanilpotency hypothesis. For every set $\pi$ of primes, the class $\mathfrak{H}_{\pi}=\left(G \in \mathfrak{S} \mid O_{\pi}(G) \leqslant Z_{\infty}(G)\right)$ is a Fischer class [2, IX.3.7b]. The intersection $\mathfrak{H}_{3} \cap \mathfrak{N}^{3}$ is a Fischer class of nilpotent length three that is not $\{2,3\}$-Hall closed.

For Fitting classes $\mathfrak{F}$ and $\mathfrak{G}$ the product

$$
\mathfrak{F G}=(G \in \mathfrak{S}: \text { there exists } N \unlhd G \text { such that } N \in \mathfrak{F} \text { and } G / N \in \mathfrak{G})
$$

is again a Fitting class (note that this is different from the Fitting class product $\mathfrak{F} \triangleright \mathfrak{G}$ ). If $\rho$ is a set of primes and $\mathfrak{F}_{r}$ a Fitting class for every $r \in \rho$, then the class $\bigcap_{r \in \rho} \mathfrak{F}(r) \mathfrak{S}_{r} \mathfrak{S}_{r^{\prime}}$ is called locally defined by the family $\{\mathfrak{F}(r) \mid r \in \rho\}$. By [2, IX.3.7c], every locally defined Fitting class is a Fischer class. It is easy to see that for Hall closed Fitting classes $\mathfrak{F}$ and $\mathfrak{G}$ the product $\mathfrak{F} \mathfrak{G}$ is also Hall closed, and that a Fitting class which is locally defined by a family of Hall closed Fitting classes is again Hall closed. But in general locally defined Fitting classes are not Hall closed:

EXAMPLE 2. Let $\mathfrak{K}$ be the supersoluble Fitting class of Example 1. Then $\mathfrak{K S}_{3} \mathfrak{S}_{3^{\prime}}$ is Hall closed, while $\mathfrak{K S}_{7} \mathfrak{S}_{7^{\prime}}$ and $\mathfrak{K S}_{7} \mathfrak{S}_{7}, \cap \mathfrak{N}^{3}$ are not Hall closed.

Proof: Let $G$ be a group in $\mathfrak{K S}_{3} \mathfrak{S}_{3^{\prime}}$. By quotient group closure of $\mathfrak{S}_{3} \mathfrak{S}_{3^{\prime}}$ we see $G / G_{\mathfrak{K}} \in \mathfrak{S}_{3} \mathfrak{S}_{3^{\prime}}$. If $H$ is a Hall- $\pi$-subgroup of $G$, then $H \cap G_{\mathfrak{\Omega}}$ is a Hall- $\pi$ subgroup of $G_{\mathfrak{K}}$, and therefore $H \cap G_{\mathfrak{K}} \in \mathfrak{K S}_{3}$. Moreover $H /\left(H \cap G_{\mathfrak{K}}\right) \cong H G_{\mathfrak{K}} / G_{\mathfrak{K}} \leqslant$ $G / G_{\mathfrak{K}} \in \mathfrak{S}_{3} \mathfrak{S}_{3^{\prime}}$. This shows $H /\left(H \cap G_{\mathfrak{K}}\right) \in \mathfrak{S}_{3} \mathfrak{S}_{3^{\prime}}$ and finally $H \in \mathfrak{K S}_{3} \mathfrak{S}_{3^{\prime}}$. Set $G=K\left\langle\langle s\rangle\right.$, where $\langle s\rangle$ is cyclic of order 7 , and $N=K_{1} \times \ldots \times K_{7}$ is the base group of $G$. Set $P_{i}=A_{i} \times B_{i}=O_{\{7,13\}}\left(K_{i}\right)$ and let $\left\langle t_{i}\right\rangle$ be a Sylow-3-subgroup of $K_{i}$. Then $U_{i}=\left\langle A_{i}, t_{i}\right\rangle$ is a Hall- $\{3,7\}$-subgroup of $K_{i}$, and $U=U_{1} \times \ldots \times U_{7}$ is a Hall- $\{3,7\}$-subgroup of $N$. Now $H=\langle U, s\rangle$ is a Hall- $\{3,7\}$-subgroup of $G$. Obviously $O_{7}(U) \leqslant H_{\mathfrak{G}}$. If $s \in H_{\mathfrak{K}}$, so $t_{1} t_{2}^{-1} \in H_{\mathfrak{K}}$, and $L=\left\langle A_{1} \times A_{2}, t_{1} t_{2}^{-1}\right\rangle$ is a subnormal subgroup of $H_{\mathfrak{K}}$. Therefore $L \in \mathfrak{K}$. On the other hand $L$ is $\{7,13\}$-perfect, a contradiction. This implies $H_{\mathfrak{K}}=O_{7}(U)$. Finally we have $H / H_{\mathfrak{K}} \cong C_{3} l C_{7} \notin \mathfrak{S}_{7} \mathfrak{S}_{7^{\prime}}$.

\section{REFERENCES}

[1] O. Brison, 'Hall operators for Fitting classes', Arch. Math. (Basel) 33 (1979), 1-9.

[2] K. Doerk and T. Hawkes, Finite soluble groups (deGruyter, Berlin, New York, 1992).

[3] M. Menth, 'A family of Fitting classes of supersoluble groups', Math. Proc. Cambridge Philos. Soc. 118 (1995), 49-57. 
[4] M. Menth, 'Examples of supersoluble Lockett sections', Bull. Austral. Math. Soc. 49 (1994), 325-332.

[5] K. Müller, 'Fittingklassen mit zusätzlichen Abschlußeigenschaften,', Arch. Math. (Basel) 50 (1988), 19-24.

\author{
Mathematisches Institut \\ Universität Würzburg \\ Am Hubland \\ D-97074 Würzburg \\ Germany
}

